\title{
Searching for Young M Dwarfs Near the Sun: Fast Rotators in the K2 Field
}

\author{
Dicy Saylor ${ }^{1}$ and Lepine ${ }^{1}$ \\ ${ }^{1}$ Georgia State University \\ email: dsaylor@astro.gsu.edu, slepine@astro.gsu.edu
}

\begin{abstract}
We present a novel way to mine the Kepler data for young M dwarfs.
\end{abstract}
Keywords. stars: rotation, methods: data analysis

\section{Introduction}

The Kepler 2 (K2) mission is targeting large numbers of nearby $\mathrm{K}$ and $\mathrm{M}$ dwarfs selected from the SUPERBLINK proper motion survey. Kepler K2 Campaign 0 and 1 monitored a total of 8607 of these cool main-sequence stars. We used the Auto-Correlation Function to search for fast rotators by examining photometric modulation in the light curves due to star spots. We identified dozens of candidate fast rotators with rotation periods less than 3 days. We investigate the possibility that they are members of nearby young moving groups. We also discuss the potential of the K2 mission to identify new nearby young $\mathrm{K} \& \mathrm{M}$ dwarfs.

\section{Methods}

We cross-matched the SUPERBLINK Catalog with the Ecliptic Plane Input Catalog (EPIC) to find high proper motion (>40mas/yr) $\mathrm{K}$ and $\mathrm{M}$ stars that were observed by Kepler K2 Campaign 0 and 1. We then ran an Auto-Correlation Function (ACF) period finding routine on those resulting 8607 stars. This ACF routine is based on the work in McQuillan et al. (2013). We preferentially selected for stars with rotation rates $0.25 d<P<4 d$, which for K-M dwarfs is typical of stars with ages $<150$ Myr (Mamajek $\&$ Hillenbrand 2008). Our search identified 46 candidate fast rotators in Campaign 0 and 214 fast rotators in Campaign 1 (due to page limit, email the authors for an electronic copy of the candidates). An example light curve and corresponding ACF is plotted in Figure 1. We estimated photometric distances for all the stars, and calculated their transverse components of motion, equivalent to $[\mathrm{V}, \mathrm{W}]$ for campaign 0 stars, which are near the Galactic anti-center, and $[\mathrm{U}, \mathrm{V}]$ for campaign 1 stars, which are near the Galactic pole.

\section{Conclusions}

Figure 2 plots the UVW space velocities of the Kepler K2 fast rotators found by this analysis. A table listing the results of this work can be supplied by the author by request. The fast rotators generally have similar kinematics to known nearby moving groups. There is a bias towards slightly lower velocities as the distances are underestimated for these young targets. Our analysis indicates that a measured fast rotation period can be an indicator of youth. Before the Kepler mission is was not feasible to measure rotation periods simply in the interest of diagnosing age. Our work implies that rotation rate 

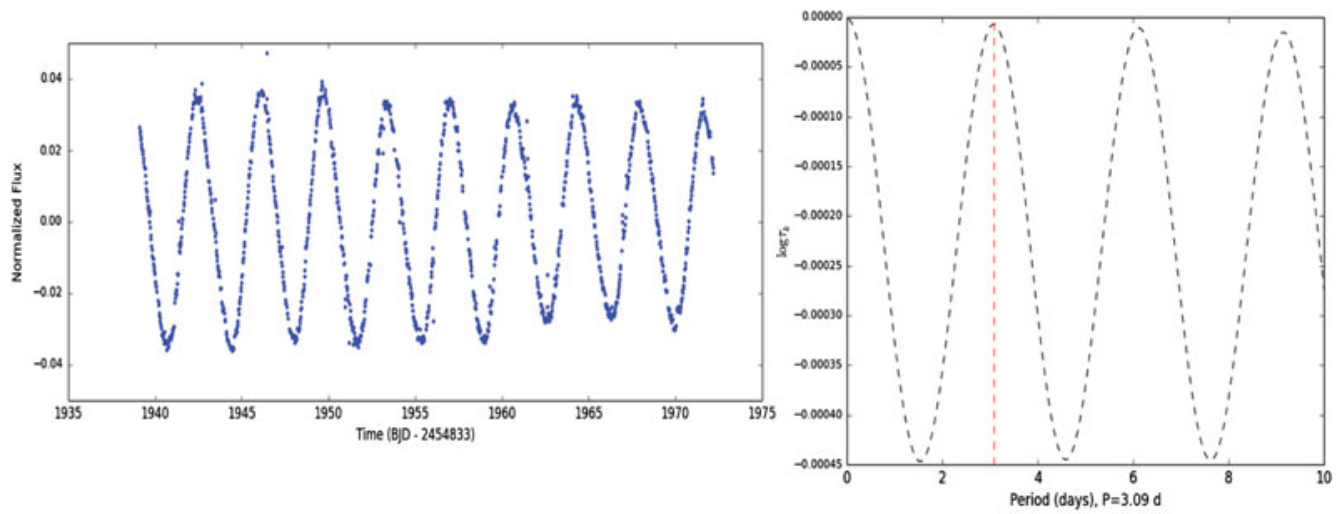

Figure 1: Example light curve of fast rotator EPIC 202068580 discovered in our search of Kepler K2 campaign 0 (left), and the corresponding auto-correlation function (right). The rotation period was calculated using an Auto-Correlation Function (ACF) developed by the authors. The resulting ACF is shown to the right of the light curve, with the detected period (3.09days) indicated by a dashed red line.
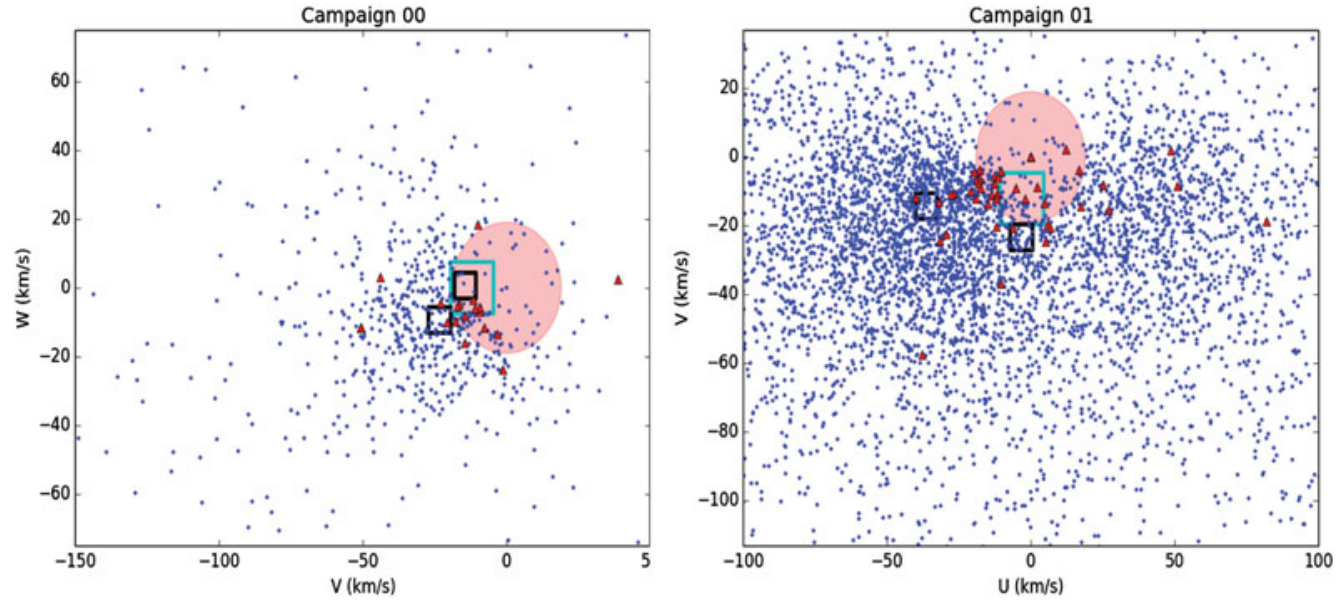

Figure 2: The derived UVW space velocities of SUPERBLINK high proper motion stars in Kepler K2 campaign 0 and 1 . The blue points are all SUPERBLINK stars in the field. The fast rotators are overplotted in red triangles. The red circle represents the incompletely sampled area of SUPERBLINK. The green square indicates the average UVW velocities of the nearby kinematic moving groups while the black squares indicate the Hyades and Pleiades motions. The SUPERBLINK fast rotators show similar space kinematics to the nearby young moving groups, indicating youth.

could be an independent indicator of youth. Future work will include searching upcoming Kepler K2 campaigns for fast rotators and computing important statistics.

\section{References}

Mamajek, E. E. \& Hillenbrand, L. A. 2008, ApJ, 687, 1264

McQuillan, A., Aigrain, S., \& Mazeh, T. 2013, MNRAS, 432, 1203 